\title{
CONDIÇÃO DE SAÚDE BUCAL DE ESTUDANTES ASSISTIDOS PELO PROGRAMA NACIONAL DE ASSISTÊNCIA ESTUDANTIL NA UFPA
}

\author{
ORAL HEALTH CONDITION OF STUDENTS ASSISTED BY THE STUDENT ASSISTANCE NATIONAL \\ PROGRAM IN UFPA
}

\author{
Ana Carolina Santiago da Silva ${ }^{a^{*}}$, Amanda Menezes Medeiros ${ }^{b^{* *}}$, \\ Dimitra Castelo Branco $^{\mathrm{c}^{* *}}$, Erick Ely Gomes de Oliveira ${ }^{\mathrm{d}^{*}}$, \\ Armando Brito Chermont ${ }^{\mathrm{e}^{*}}$, Liliane Silva do Nascimento ${ }^{\mathrm{f}^{*}}$

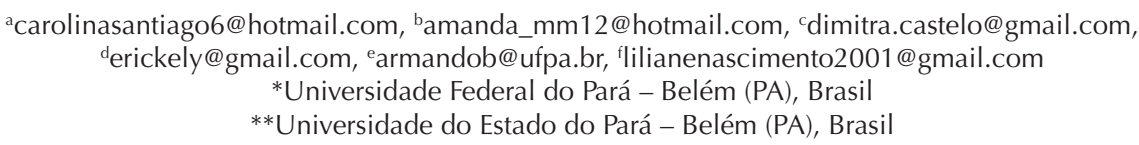

Data de recebimento do artigo: 25/09/2017

Data de aceite do artigo: 25/10/20177

\section{RESUMO}

Introduçáo: A política de saúde estudantil contempla o cuidado integral e visa garantir melhores condiçôes de saúde para a população universitária. Instituiçôes públicas de ensino superior desenvolvem projetos de cuidado com a saúde de modo a favorecer a convivência e a aprendizagem dos estudantes. Objetivo: $\mathrm{O}$ objetivo deste trabalho foi avaliar a condição da saúde bucal dos estudantes assistidos pelo Programa Nacional de Assistência Estudantil (Pnaes). Materiais e métodos: A pesquisa foi desenvolvida na Universidade Federal do Pará por meio de análise da saúde bucal de usuários de projetos de atendimento odontológico. A amostra foi constituída de 250 estudantes dos cursos de graduação no campus de Belém. A coleta de dados foi feita por meio de exame clínico bucal e preenchimento de questionário; esses dados sofreram análise estatística a partir dos softwares BioEstat 5.0 e R 3.3.1. Resultados: O perfil desse estudante usuário do Pnaes é predominantemente de mulher, brasileira, solteira, sem filhos, que mora com os pais e possui renda familiar de até 3 salários mínimos; o CPO-D médio encontrado foi de 8,3; 74,4\% dos avaliados apresentaram sangramento gengival, cálculo dentário e/ou bolsa periodontal, e 97,6\% buscam hospitais públicos quando adoecem. Conclusão: A maioria dos estudantes apresentam algum tipo de incômodo relacionado aos dentes e/ou à boca, além de estarem insatisfeitos com sua condiçáo de saúde bucal. A observação da saúde bucal desses estudantes permite que a instituição conheça o perfil dos usuários de projetos de assistência e elabore políticas internas voltadas para atender, de forma mais efetiva, seus estudantes em condição de vulnerabilidade.

Palavras chave: Assistência odontológica integral; assistência à saúde; assistência integral à saúde; políticas públicas de saúde; saúde bucal.

\section{ABSTRACT}

Introduction: The students health policy includes comprehensive care and aims to ensure better health for the university population. Public higher education institutions develop health care projects in order to promote students' interaction and learning. Objective: The objective of this study was to evaluate the oral health status of students assisted by the Student Assistance National Program (PNAES). Materials and methods: The research was conducted at Universidade Federal do Pará and it analyzed the oral health of dental care projects' users. The sample consisted of 250 undergraduate students of Belém campus. Data collection was made by means of oral clinical examination and questionnaire filling; this data underwent statistical analysis with BioEstat software 5.0 and R 3.3.1. Results: The students who are PNAES users are predominantly female, Brazilian, single, who have no children, live with their parents and have a 
family income of up to 3 minimum wages; the average DFMS found was $8.3 ; 74.4 \%$ of the individuals presented gingival bleeding, dental calculus and/or periodontal pocket, and $97.6 \%$ of them look for public hospitals when they get sick. Conclusion: Most students presented some dental or mouth discomfort and were dissatisfied about their oral health condition. The awareness of oral health status of these students allows the institution to know the profile of assistance projects users and to develop internal policies geared to assist their students in a vulnerable condition more effectively.

Keywords: Comprehensive dental care; delivery of health care; comprehensive health care; public health policies; oral health.

\section{Introdução}

Nas últimas décadas, instituições multilaterais, como a Organização Mundial da Saúde (OMS) e a Organização Pan-americana de Saúde (Opas), governos nacionais, regionais e locais e organizaçóes do terceiro setor, têm disseminado o conceito e apoiado a estratégia de criação de ambientes saudáveis ${ }^{1,2}$.

O desenvolvimento de projetos de promoção de saúde dentro do espaço das universidades tem sido reconhecido como uma alternativa intersetorial viável, que resulta em melhores processos formativos e na melhoria da qualidade de vida de uma importante parcela da populaçáo. Especificamente, ações promocionais voltadas para estudantes favorecem uma formação integral, estimulando a prática profissional responsável e engajada com a realidade social ${ }^{3}$.

A busca da redução das desigualdades socioeconômicas faz parte do processo de democratização da universidade e da própria sociedade brasileira. Essa democratização náo se pode efetivar apenas mediante o acesso à educação superior gratuita; torna-se, então, necessário a criação de mecanismos que garantam a permanência dos ingressantes, reduzindo os efeitos das desigualdades apresentadas por um conjunto de estudantes provenientes de segmentos sociais cada vez mais pauperizados, que apresentam dificuldades concretas de prosseguir sua vida acadêmica com sucesso ${ }^{4}$.

O Programa Nacional de Assistência Estudantil (Pnaes) é uma das açóes advindas do Plano de Desenvolvimento da Educação (PDE), elaborado e implantado em 2008 com objetivo de atender aos discentes matriculados em cursos de graduação presencial das instituições federais de ensino superior (IFES), visando promover o apoio à permanência e à conclusão dos estudos dos estudantes de baixa condição socioeconômica 5 .

O programa contempla açóes de assistência estudantil desenvolvidas nas seguintes áreas: moradia estudantil; alimentação; transporte; assistência à saúde; inclusão digital; cultura; esporte; creche; e apoio pedagógico. As açôes de assistência estudantil sugeridas pelo Pnaes priorizam viabilizar a igualdade de oportunidades e contribuir para o melhoramento do desempenho acadêmico, além de agir, preventivamente, para minimizar as situaçóes de reprovação e evasão oriundas da baixa condição financeira do estudante. Através do repasse de recursos, as IFES implantam as açóes e definem os critérios de seleção dos estudantes que serão beneficiados pelos projetos ${ }^{5,6}$.

É necessário a priorização da saúde da comunidade universitária pelas instituiçóes de ensino superior, na lógica de criar ambientes de trabalho, de aprendizagem e de sociabilidade saudáveis ligados à promoção de práticas de saúde que implicariam uma melhor formação para esses futuros profissionais 7 . Sendo assim, é extremamente relevante o envolvimento das universidades, pois, além dos benefícios citados anteriormente, a imagem pública da instituiçáo passa a ser valorizada e pode mostrar, nos níveis local, regional e nacional, a importância do incentivo à promoção da saúde 8 .

$\mathrm{Na}$ literatura encontram-se trabalhos relacionados aos fatores de risco para a saúde de estudantes universitários, ressaltando-se, entre eles, a alimentação inadequada, a ausência de atividades físicas, o consumo de drogas ilícitas, entre outros ${ }^{9,10}$. Entre os diversos agravos que esse grupo pode sofrer, destaca-se o aspecto de saúde bucal que, como já demonstram alguns estudos, pode impactar o desempenho das funçôes diárias e provocar insuficiência do rendimento acadêmico ${ }^{11,12}$. A saúde bucal de uma população está intimamente relacionada às suas condições sociais, econômicas e culturais, bem como ao acesso e ao cuidado com a saúde ${ }^{13}$. Dessa maneira, esta pesquisa visa analisar as condiçóes de saúde bucal dos estudantes assistidos pelo Pnaes de uma universidade.

\section{Metodologia}

Realizou-se estudo do tipo transversal na Universidade Federal do Pará (UFPA), com prévia aprovação do Comitê de Ética em Pesquisa Envolvendo seres Humanos do Instituto de Ciências da Saúde da UFPA, de acordo com a Resolução no 466/12 e suas complementares do Conselho Nacional de Saúde14,15, sob o parecer de número 1.669.201. 
A instituição é uma das maiores e mais importantes da Amazônia, com 32.169 alunos matriculados nos cursos de graduação. O principal campus está localizado na cidade de Belém. No atual sistema de ingresso na universidade, uma parte das vagas é destinada para o sistema de cotas, no qual a UFPA reserva $50 \%$, no mínimo, das vagas de cada curso e turno para candidatos que comprovarem ter cursado todo o ensino médio em escola pública, divididas igualmente entre candidatos oriundos de famílias com renda igual ou inferior a 1,5 salário mínimo per capita e os com renda superior a 1,5 salário mínimo per capita. Em cada um desses dois grupos, $78 \%$ das vagas são destinadas a autodeclarados pretos, pardos ou indígenas ${ }^{16}$.

$\mathrm{Na}$ condução desta pesquisa considerou-se trabalhar o termo vulnerabilidade. Esse termo relaciona-se à ideia de procurar compreender um conjunto de elementos que caracterizam as possibilidades e condiçóes de vida de uma pessoa ou de um grupo, bem como avaliar em que medida essas pessoas têm acesso a serviços. Vulnerabilidade representa não apenas uma nova forma de expressar um problema bem estabelecido, mas também uma busca da superaçáo de preconceitos por meio da construção de uma nova mentalidade, uma nova maneira de perceber e tratar os grupos sociais e avaliar suas condiçóes de vida, proteção social e segurança. É uma busca por mudança no modo de encarar as populaçóes-alvo dos programas sociais $s^{17,18}$.

A amostra desse trabalho foi composta de 250 estudantes da UFPA atendidos em projetos de assistência odontológica. O encaminhamento para tratamento parte da Diretoria de Assistência e Integração Estudantil da Pró-Reitoria de Extensão (Daie/Proex) para os serviços de referência, e o atendimento ocorre em horários agendados. Foram considerados como critérios de inclusão de indivíduos: estar devidamente matriculado em qualquer curso da universidade e apresentar o encaminhamento para assistência. As coletas dos dados foram realizadas no período da manhã e da tarde, durante os horários estabelecidos para o funcionamento dos projetos de assistência entre maio de 2015 e maio de 2016.

Os voluntários foram escolhidos de acordo com a ordem de procura por atendimento odontológico e a aceitaçáo em participar da pesquisa, respeitando-se os critérios de inclusão. A obtenção dos dados foi realizada após assinatura do termo de consentimento livre e esclarecido (TCLE) e preenchimento do questionário especialmente desenvolvido para a pesquisa, com parte das questóes adaptadas do questionário do Projeto SB Brasil ${ }^{19}$. Exames clínicos individuais foram realizados por duas acadêmicas devidamente calibradas para aferição dos índices de condição dentária (índice CPO-D) e condição periodontal.

Condutas adequadas de biossegurança foram mantidas em todas as consultas. A equipe fez uso de equipamentos de proteção individual e nos pacientes foram colocados capôs descartáveis. Os kits clínicos para exame intraoral eram compostos por pinça clínica, sonda periodontal da OMS (instrumento padronizado pela OMS), sonda exploradora e espelho clínico, os quais foram devidamente esterilizados para utilização.

$\mathrm{Na}$ análise dos dados foram obtidas distribuições absolutas e as medidas estatísticas média, mediana e moda (estatística descritiva), tendo-se utilizado o teste estatístico t-Student para duas amostras independentes, considerando-se o nível de significância de $1 \%(\mathrm{p} \leq 0,01)$. Utilizou-se o teste de ShapiroWilk para verificar a normalidade na distribuição dos dados, adotando-se nível de significância de 1\% $(\mathrm{p} \leq 0,01)$. Os dados foram analisados nos softwares BioEstat 5.0 e R 3.3.1.

\section{Resultado}

Dos 250 estudantes atendidos observou-se que $60 \%(n=150)$ eram do sexo feminino e $40 \%$ $(\mathrm{n}=100)$, do sexo masculino. Dessa amostra total, $94,4 \%$ se declararam solteiros e $5,6 \%$ casados ou separados. A maioria $(96,8 \%)$ possui residência em zona urbana, e o restante, em zona rural (3,2\%); $98,8 \%$ não são portadores de necessidades especiais, e apenas 1,2\% afirmaram ter algum tipo de deficiência. $95,2 \%$ dos analisados ainda não possuem filhos. A renda familiar mensal de $86 \%$ dos estudantes entrevistados era de até três salários mínimos. Observouse na distribuiçáo percentual para cada instituto da UFPA o maior número de atendidos e a média do índice de CPO-D dos estudantes (Tabela 1). A maioria dos usuários do projeto era matriculada no Instituto de Ciências da Saúde (ICS) e no Instituto de Ciências Sociais Aplicadas (ICSA).

Tabela 1: Distribuição dos estudantes assistidos pelo Pnaes na UFPA em relação ao índice CPO-D médio, por instituto de en$\operatorname{sino}(n=250)$.

\begin{tabular}{llcc} 
Instituto & $\mathbf{N}$ & F\%* & Média do CPO-D \\
\hline ICS & 50 & 20.00 & 7,88 \\
ICEN & 45 & 18.00 & 8,38 \\
ICSA & 39 & 15.60 & 8,54 \\
\hline
\end{tabular}

continua... 
Tabela 1: Continuação.

\begin{tabular}{lccc}
\hline Instituto & $\mathbf{N}$ & $\mathbf{F \% *}$ & Média do CPO-D \\
\hline ITEC & 32 & 12.80 & 8,06 \\
ICED & 23 & 9.20 & 7,52 \\
\hline ILC & 20 & 8.00 & 8,95 \\
IFCH & 18 & 7.20 & 9,33 \\
ICJ & 8 & 3.20 & 8,50 \\
ICA & 6 & 2.40 & 9,17 \\
IG & 5 & 2.00 & 7,00 \\
IEMCI & 3 & 1.20 & 10,67 \\
ICB & 1 & 0.40 & 3,00 \\
Total & 250 & 100.00 & 8,3 \\
\hline
\end{tabular}

*Frequência em porcentagem

Fonte: Elaborado pelos autores, 2017

Descriçãa: Instituto de Ciências da Saúde (ICS), Instituto de Ciências Exatas e Naturais (ICEN), Instituto de Ciências Sociais Aplicadas (ICSA), Instituto de Tecnologia (ITEC), Instituto de Educação (ICED), Instituto de Letras e Comunicação (ILC), Instituto de Filosofia e Ciências Humanas (IFCH), Instituto de Ciências Jurídicas (ICJ), Instituto de Ciências da Arte (ICA), Instituto de Geociências (IG), Instituto de Educação Matemática e Científica (IEMCI), Instituto de Ciências Biológicas (ICB).

Com relação aos tipos de serviços de saúde procurados pelos estudantes quando adoecem, verificou-se que $97,6 \%$ recorrem aos hospitais públicos do Sistema Único de Saúde (SUS). Quanto à autoavaliação de saúde bucal, a grande maioria $(99,2 \%)$ concordou que precisa de algum tratamento dentário atualmente, e 84,8\% queixaram-se de ter tido dor de dente nos últimos seis meses. Dos estudantes avaliados, $2,4 \%$ relataram nunca ter ido ao dentista, $44 \%$ foram ao dentista há menos de um ano, $27,6 \%$ foram de um a dois anos atrás, $23,2 \%$ não vão ao dentista há três anos ou mais, e os outros $5,2 \%$ não souberam responder ou não responderam à pergunta. Podemos observar que uma parte significativa $(69,2 \%)$ dos entrevistados foi atendida pela última vez por dentista do serviço público (SUS).

$\mathrm{Na}$ análise do grau de satisfação dos estudantes com seus dentes e boca encontrou-se que: $52 \%$ estavam insatisfeitos; $25,2 \%$ não estavam satisfeitos nem insatisfeitos; $20 \%$ estavam muito insatisfeitos; apenas 2,4\% relataram satisfação; e $0,4 \%$ não soube responder ou não respondeu. Dessa maneira, pode-se analisar que mais de $70 \%$ dos estudantes atendidos pelo projeto buscaram atendimento devido à insatisfação quanto à sua saúde bucal.

Obteve-se como média, moda e mediana do índice CPO-D os seguintes valores, respectivamente: 8,3; 9 e 8. Verificou-se a distribuição normal dos dados relacionados ao índice CPO-D tanto para estudantes do sexo masculino ( $p$-valor $=0,2312)$ como para os do sexo feminino ( $\mathrm{p}$-valor $=0,09806)$.
Para a avaliaçâo da existência de diferença estatística no índice CPO-D entre o sexo feminino e masculino foi utilizado teste t-Student paramétrico para duas amostras independentes. Considerou-se como hipótese nula que em média o índice CPO-D entre os estudantes de sexo feminino é igual ao índice CPO-D dos estudantes do sexo masculino, e como hipótese alternativa que em média o índice CPO-D entre os estudantes do sexo feminino é diferente do índice CPO-D dos estudantes do sexo masculino. Obteve-se ; logo pode-se concluir que o índice CPO-D dos estudantes independe do sexo. Analisando-se o intervalo de confiança [-1,572120; $0,338787]$, podemos verificar que o zero está contido nesse intervalo, o que reforça a hipótese de igualdade das médias entre os dois grupos.

A condição periodontal desses estudantes foi analisada pela ausência ou presença de sangramento gengival, cálculo dentário e bolsa periodontal (Tabela 2). Verificou-se que $25,6 \%$ não apresentaram nenhum tipo de alteração periodontal, 25,2\% possuíam uma das variáveis, $18 \%$ apresentaram duas, e $31,20 \%$ apresentaram as três variáveis.

Tabela 2: Distribuição dos estudantes assistidos pelo Pnaes na UFPA em relação aos tipos de alteração periodontal $(n=250)$.

\begin{tabular}{lcc} 
Alteração periodontal & N & F\%* \\
$\begin{array}{l}\text { Sangramento, cálculo dentário e bolsa } \\
\text { periodontal }\end{array}$ & 78 & 31.20 \\
Cálculo dentário & 48 & 19.20 \\
Sangramento e cálculo dentário & 31 & 12.40 \\
Sangramento & 15 & 6.00 \\
Cálculo dentário e bolsa periodontal & 7 & 2.80 \\
Sangramento e bolsa periodontal & 7 & 2.80 \\
Nenhum & 64 & 25.60 \\
Total & 250 & 100.00 \\
\hline
\end{tabular}

${ }^{*}$ Frequência em porcentagem

Fonte: Elaborado pelos autores, 2017.

Sobre a morbidade referida, $40,8 \%$ dos estudantes afirmaram ter dificuldade para comer por causa dos dentes ou ter sentido sensibilidade ao ingerir líquidos gelados ou quentes; $43,6 \%$ disseram que seus dentes incomodavam durante a escovação; $25,6 \%$ disseram que seus dentes os(as) deixam nervosos(as); 12,8\% já deixaram de sair e $23,2 \%$ já deixaram de praticar esportes em razáo de problemas com os dentes; $12,8 \%$ já tiveram dificuldade para falar por conta dos dentes; $33,2 \%$ disseram que seus dentes o(a) atrapalham para estudar, trabalhar ou fazer alguma tarefa da faculdade; e 61,6\% disseram que já deixaram de dormir ou dormiram mal por causa dos dentes.

Quanto ao conhecimento da Pnaes, 61,6\% relataram não compreendê-la nas instituições de ensino superior. 
Entre os que não conheciam tal política e alguns que já a conheciam, 67,6\% (121 estudantes) expuseram que falta maior divulgação desta pelas instituições de ensino superior; $25,1 \%$ (45 estudantes) disseram que faltam visitas da equipe técnica da Daie/Proex aos campi para reuniōes esclarecedoras com a comunidade universitária; e 7,2\% (4 estudantes) disseram que falta informação em manuais. Dos estudantes que participaram da pesquisa, 43,6\% eram assistidos por algum programa de assistência estudantil, e $84,4 \%$ já foram atendidos por projetos de assistência estudantil na UFPA.

\section{Discussão}

Este estudo mostrou que apesar de o Pnaes existir desde 2008, grande parcela dos estudantes atendidos não compreende a finalidade da política de assistência estudantil dentro das instituiçóes de ensino superior ${ }^{20}$. A divulgação insuficiente para a comunidade universitária foi identificada, apesar de mais da metade dos componentes da amostra serem usuários de algum projeto de assistência estudantil dentro da universidade. $\mathrm{O}$ perfil desse estudante usuário do Pnaes é predominantemente de mulher, brasileira, solteira, sem filhos, residente em zona urbana, que utiliza o transporte coletivo, mora com os pais, possui casa própria e renda familiar de até três salários mínimos.

Observa-se que a maioria dos estudos epidemiológicos segue os parâmetros preconizados pela OMS21 e as idades-índice não contemplam a faixa etária de 19 a 35 anos, exatamente na qual os estudantes avaliados se enquadram - predominantemente adultos jovens. Dessa maneira, torna-se difícil compararmos a condição de saúde bucal e periodontal desses estudantes às médias nacionais. Porém, se fizermos uma média do índice CPO-D do último levantamento SB Brasil ${ }^{19}$, que foi de 4,25 dentes em adolescentes de 15 a 19 anos e 16,7 dentes em adultos de 35 a 44 anos, teríamos como média 10,47; ou seja, se levarmos em consideração a média 8,3 de CPO-D obtida na pesquisa, a condição de saúde bucal dos estudantes está próxima à média nacional.

No que tange à condição periodontal, destaca-se que $74,4 \%$ dos avaliados apresentaram sangramento gengival, cálculo dentário e/ou bolsa periodontal, que são indicativos de gengivite. Nesse sentido, se esses estudantes não tiverem, em longo prazo, assistência odontológica, orientaçóes e hábitos de higiene oral adequados, possuem alto risco para desenvolvimento de doença periodontal ${ }^{22}$. A gengivite é a forma mais comum e prevalente de doença periodontal em crianças e adolescentes, que pode se iniciar na infância e na adolescência e progredir lentamente durante toda a vida23. Considerando-se o caráter cumulativo de destruição dos tecidos periodontais, torna-se relevante a compreensão das suas características epidemiológicas e clínicas no início do curso da doença. Esses dados devem servir de base para intervençóes apropriadas, voltadas à prevenção da sua progressão e ao seu tratamento precoce ${ }^{24,25}$.

Observou-se ainda que os estudantes sofreram algum tipo de incômodo relacionado à boca e/ou aos dentes. Notou-se que o grau de insatisfação é alto entre esses pacientes, e a maioria deles não está satisfeita com a atual condição de saúde bucal. Dessa maneira, em consonância com outros estudos desenvolvidos, pode-se perceber que problemas dentários interferem de forma negativa no dia-a-dia ${ }^{26,27}$ desses estudantes, haja vista que relataram ter dificuldades para consumir líquidos gelados ou quentes devido à sensibilidade, para mastigar, para falar e até mesmo para dormir em decorrência de agravos dentários. Isso influencia não apenas a saúde bucal, mas abrange problemas de autoestima e psicológicos, interferindo na sua qualidade de vida e no rendimento escolar ${ }^{11,12}$, visto que $33,2 \%$ afirmaram que já tiveram dificuldade para realizar tarefas da faculdade ou do trabalho devido a problemas de origem bucal.

No que se relaciona à autopercepção da saúde bucal, a porcentagem de estudantes que afirmaram necessitar de tratamento foi alta, o que foi confirmado pela avaliação clínica. Além disso, a insatisfação com a saúde bucal foi manifestada por mais da metade da amostra, e uma parcela significativa havia tido dor de dente nos seis meses anteriores à pesquisa. Esses achados estão em consonância com os resultados da pesquisa nacional de saúde bucal de $2010^{19}$, bem como com o que foi encontrado em pesquisa relacionada ${ }^{13}$, que acrescenta outros impactos prevalentes associados à condição de saúde bucal, como: dificuldade para comer; incômodo para escovar os dentes; irritabilidade ou nervosismo e vergonha ao sorrir.

\section{Conclusão}

A observação da condição de saúde bucal dos usuários de projetos de assistência estudantil permite que a instituição conheça o perfil desses estudantes em vulnerabilidade, elabore políticas internas para atendê-los de forma mais efetiva e reconheça a importância da reformulação e implementação de políticas públicas educacionais e de assistência que deem suporte à correção das profundas distorçôes socioeconômicas relacionadas à origem dos alunos que lograram acesso ao ensino superior. Leve-se em conta o Pnaes como componente de 
uma política pública focalizada, destinada a assegurar a manutenção e a conclusão do curso nas IFES pelos graduandos que se encontram em situação de fragilidade socioeconômica.

No que tange à saúde bucal, os estudantes de ambos os sexos apresentaram uma condiçáo razoável, verificando-se a necessidade de acompanhamento odontológico tanto para prevenir agravos quanto para tratar os já existentes, de forma que alcancem uma melhor qualidade de vida e desempenho escolar. Verificou-se que a maioria dos estudantes com renda de até três salários mínimos, menor renda considerada neste estudo, estavam insatisfeitos em relação à sua condição de saúde bucal e já haviam apresentando algum incômodo nos dentes e/ou na boca. Dessa maneira, considerando-se que os estudantes avaliados nesta pesquisa estão em vulnerabilidade socioeconômica, podemos sugerir que os agravos em saúde bucal avaliados podem ter relação com suas condiçóes de vida, bem como podem gerar consequências que interfiram negativamente no cotidiano desses universitários, principalmente no âmbito do curso de graduação.

Deve-se atentar para a importância de ampliar serviços eficazes e resolutivos, já que o estudante passa boa parte do seu tempo nas IFES, como também fortalecer os cenários de aprendizagem e trabalho intersetorial.

Apesar de este estudo possuir limitaçóes metodológicas, e os resultados precisarem ser ainda explanados em futuros trabalhos, há que se subsidiar maior literatura acerca do tema. Contudo, pode-se analisar a condiçáo de saúde bucal desses estudantes e dessa forma traçar seu perfil, buscando com isso contribuir para minimizar a lacuna etária dos levantamentos epidemiológicos nacionais.

\section{Referências}

1. Organização Pan-Americana da Saúde. Divisão de Promoção e Proteção da Saúde. Municípios e comunidades saudáveis. Guia dos prefeitos para promover qualidade de vida. Washington, DC: OPAS; 2003. 74 p.

2. World Health Organization. Health-Promoting Schools: a healthy setting for living, learning and working. Geneva: WHO; 1998. 12 p.

3. Tsouros AD, Dowding G, Thompson J, Dooris M (Ed.). Health promoting universities: concept, experience and framework for action. Copenhagen: WHO Regional Office for Europe; 1998. 174 p.

4. Alves JM. A assistência estudantil no âmbito da política de Ensino Superior Pública. Serv Soc Rev. 2002 jul/dez [citado em 2015 ago 28];5(1):408-22. Disponível em: https://goo.gl/MXDSrk
5. Vasconcelos NB. Programa nacional de assistência estudantil: uma análise da evolução da assistência estudantil ao longo da história da educação superior no Brasil. Ens Rev [Internet]. $2010 \mathrm{jul} / \mathrm{dez}$ [citado em 2015 nov 2];17(2):599-615. Disponível em: https://goo.gl/qxEPBj

6. Brasil. Decreto no 7.234, de 19 julho de 2010. Diário Oficial da União [Internet]. 2010 jul 20 [citado em 2015 set 1];1:5. Disponível em: https://goo.gl/CN8ahJ

7. Mello ALSF, Moysés ST, Moysés SJ. A universidade promotora de saúde e as mudanças na formação profissional. Interface Comun Saúde e Educ [Internet]. 2010 set. [citado em 2015 ago 28];14(34):683-92. Disponível em: https://goo.gl/MJbZhV

8. Mota LQ, Santos TA, Magalhães DBL. Humanização no atendimento odontológico: acolhimento da subjetividade dos pacientes atendidos por alunos de graduação nos campos de estágio. Rev Bras Ciênc Saúde [Internet]. 2012 [citado em 2015 ago 31];16(4):537-44. Disponível em: https://goo.gl/fk6nXZ

9. Haddad LG, Malak MZ. Smoking habits and attitudes towards smoking among university students in Jordan. Int J Nurs Stud. 2002 Nov;39(8):793-802.

10. Khami MR, Murtomaa H, Razeghi S, Virtanen JI. Smoking and its determinants among Iranian dental students. Med Princ Pract. 2010 jul;19(5):390-4.

11. Arévalo SJ, Rivera MF, Rivera IC, Sánchez F. Situación de la salud bucal de la población universitária hondureña. Rev Med Hondur [Internet]. 2005 [citado em 2015 jun 5];73(4):161-5. Disponível em: https://goo.gl/qXU2EY

12. Pinto SCS, Alferes-Araújo CS, Wambier DS, Pilatti GL, Santos FA. Oral hygiene habits among undergraduate university students. Pesqui Bras Odontopediatria Clín Integr [Internet]. 2008 jul/ago [citado em 2015 jul 5];8(3):353-8. Disponível em: https://goo.gl/M8UJ6b

13. Freire MCM, Martins AB, Santos CR, Martins NO, Filizzola EM, Jordão LMR, et al. Condição de saúde bucal, comportamentos, autopercepção e impactos associados em estudantes universitários moradores de residências estudantis. Rev Odontol UNESP [Internet]. 2012 maio/ jun [citado em 2015 jul 5];41(3):185-91. Disponível em: https://goo.gl/xD62oN

14. Brasil. Ministério da Saúde. Conselho Nacional de Saúde. Resolução no 466, de 12 de dezembro de 2012. Diário Oficial da União [Internet]. 2013 jun 13 [citado em 2016 ago 15];1:59. Disponível em: https://goo.gl/WA1X8c

15. Brasil. Ministério da Saúde. Conselho Nacional de Saúde. Norma Operacional no 001/2013. Brasília, DF: Conselho Nacional de Saúde; 2013 [citado em 2016 ago 15]. Disponível em: https://goo.gl/HnsUoh

16 Universidade Federal do Pará. Histórico estrutura[Internet]. Belém: UFPA; c2012-2015 [citado em 2016 ago 20]. Disponível em: https://goo.gl/Bpmab5 
17. Lessick M, Woodring BC, Naber S, Halstead L. Vulnerability: a conceptual model applied to perinatal and neonatal nursing. J Perinat Neonatal Nurs. 1992 dez; 6(3):1-14.

18. Adorno, RCF. Capacitaçáo solidária: um olhar sobre os jovens e sua vulnerabilidade social. la ed. São Paulo: Associação de Apoio ao Programa Capacitação Solidária; 2001.

19. Brasil. Ministério da Saúde. Secretaria de Atenção à Saúde. Departamento de Atenção Básica. Coordenação Nacional de Saúde Bucal. Projeto SB Brasil 2010: condições de saúde bucal da população brasileira em 2010: resultados principais. Brasília, DF: Ministério da Saúde; 2011.

20. Silveira MM. A assistência estudantil no ensino superior: uma análise sobre as políticas de permanência das universidades federais brasileiras [dissertação]. Pelotas (RS): Universidade Católica de Pelotas; 2012.

21. World Health Organization. Oral health surveys: basic methods. $5^{\mathrm{a}}$ ed. Geneva: WHO; 2013.

22. Souza CHC, Dantas-Neta NB, Laurentino JB, Nunes-dosSantos DL, Prado Júnior RR, Mendes RF. Fatores de risco relacionados à condição de saúde periodontal em universitários. Rev Odontol UNESP [Internet]. 2013 maio/jun [citado em 2015 jul 20];42(3):152-59. Disponível em: https://goo.gl/2T7YZG
23. Califano JV, Research Science and Therapy Committee American Academy of Periodontology. Position paper: periodontal diseases of children and adolescents. J Periodontol. 2003 Nov;74(11):1696-704.

24. Collins J, Carpio AM, Bobadilla M, Reyes R, Gúzman I, Martínez B, et al. Prevalence of clinical attachment loss in adolescents in Santo Domingo, Dominican Republic. J Periodontol. 2005 set;76(9):1450-4.

25. Igic M, Kesic L, Lekovic V, Apostolovic M, Mihailovic D, Kostadinovic L, et al. Chronic gingivitis: the prevalence of periodontopathogens and therapy efficiency. Eur J Clin Microbiol Infect Dis [Internet]. 2012 ago [citado em 2015 ago 5];31(8):1911-5. Disponível em: https://goo.gl/tXVfsu

26. Pereira AL. Influência da condição de saúde bucal na qualidade de vida dos indivíduos [trabalho de conclusão de curso]. Campos Gerais (MG): Universidade Federal de Minas Gerais; 2010 [citado em 2016 ago 16].

27. Ramalho LEG. Abordagem avaliativa da política de assistência estudantil em uma instituição de ensino profissional [dissertação]. Juiz de Fora (MG): Universidade Federal de Juiz de Fora; 2013 [citado em 2015 ago 20].

\section{Como citar este artigo:}

